\title{
Unravelling the therapeutic potential of IL-33 for atrophic AMD
}

\author{
Alison J. Clare (D) ${ }^{1}$, Jian Liu ${ }^{1}$, David A. Copland ${ }^{1}$, Sofia Theodoropoulou ${ }^{1}$ and Andrew D. Dick (D) ${ }^{1,2,3,4 凶}$ \\ (c) The Author(s) 2021
}

\begin{abstract}
Age-related macular degeneration (AMD), a degenerative disease affecting the retinal pigment epithelium (RPE) and photoreceptors in the macula, is the leading cause of central blindness in the elderly. AMD progresses to advanced stages of the disease, atrophic AMD (aAMD), or in 15\% of cases "wet" or neovascular AMD (nAMD), associated with substantial vision loss. Whilst there has been advancement in therapies treating $n A M D$, to date, there are no licenced effective treatments for the $85 \%$ affected by aAMD, with disease managed by changes to diet, vitamin supplements, and regular monitoring. AMD has a complex pathogenesis, involving highly integrated and common age-related disease pathways, including dysregulated complement/ inflammation, impaired autophagy, and oxidative stress. The intricacy of AMD pathogenesis makes therapeutic development challenging and identifying a target that combats the converging disease pathways is essential to provide a globally effective treatment. Interleukin-33 is a cytokine, classically known for the proinflammatory role it plays in allergic disease. Recent evidence across degenerative and inflammatory disease conditions reveals a diverse immune-modulatory role for IL-33, with promising therapeutic potential. Here, we will review IL-33 function in disease and discuss the future potential for this homeostatic cytokine in treating AMD.
\end{abstract}

Eye (2022) 36:266-272; https://doi.org/10.1038/s41433-021-01725-5

\section{INTRODUCTION}

Age-related macular degeneration (AMD) is the leading cause of blindness among over-65s in developed countries and accounts for $8.7 \%$ of all blindness worldwide [1, 2]. With an ever-growing aging population, AMD has become an increasing health problem. Characterised by the progressive loss in the macular region of specialised cells, retinal pigment epithelium (RPE), and photoreceptors, AMD can progress into two advanced forms the "neovascular"-wet form (nAMD) and for the majority of patients the "atrophic"-dry form (aAMD). Whilst later stages of nAMD can, to various degrees, be successfully treated with VEGF-blocking agents, treatments to target early in the disease or tackle advancement to neovascularisation or late-stage aAMD are still lacking [2-6]. With the disease projected to affect 288 million people globally by 2040 [1], the unmet need for an effective therapeutic in early AMD is pressing.

Age is the leading risk factor for AMD and the aetiology is multifactorial. Data implicates the presence of chronic low-grade inflammation in the pathogenesis of AMD but the underlying causes and mechanisms responsible for persistent inflammatory responses are not fully appreciated. Environmental risk factors, including diet, obesity, light exposure, and smoking, alongside susceptibility genes, increase the risk of developing and are associated with greater severity of disease [7]. Multiple genomewide association studies have identified risk loci for AMD. For example, strong associations are seen with complement pathway and ARMS2 alleles, which denote a large increase in disease risk, and identification of very rare coding variants in CFI, CFH, and TIMP3 suggests causal roles for these genes $[8,9]$. A more recent study has teased out risk variants in early vs. late AMD, identifying variants in pathways affecting complement complex and lipid metabolism as significant contributors in early and advanced AMD, with extracellular matrix metabolism identified among advanced-AMD risk loci only [10]. Understanding of genetic risk has driven approaches to developing therapeutics, including inhibitors and gene therapies targeting complement pathway [11-13]. However, the low penetrance of such genetic risk factors $[8,9]$ has led to mixed success with the greatest promise seen only for the small number of patients with relevant risk variants [11]. The disease complexity and involvement of converging pathways are supported as mice with complement perturbation do not fully emulate AMD pathology [14-16]. In addition to immune-mediated inflammation, pathways implicated in AMD include oxidative stress, mitochondrial dysfunction, lipid metabolism, autophagy, and cellular senescence $[4-6,17]$, all of which are interrelated and exert a cause-and-effect on each other; a vicious cycle ultimately leading to retinal degeneration. These immune, metabolic, and tissue responses occurring in AMD and retinal degeneration involve the integrated function of multiple cell types (such as RPE, microglia, choroidal endothelial, macrophages, and mast cells as well as infiltrating immune cells), and a combination of several genetic and environmental factors [2, 5, 6, 18]. Targeting therapeutic approaches that modulate cellular biogenetics and immune pathways may serve to protect the normal cell and tissue health, reaching a wider patient population.

The early stages of AMD are clinically defined by the presence of small drusen deposits and pathological changes to the RPE [19]. Located and notwithstanding presence of subretinal drusenoid

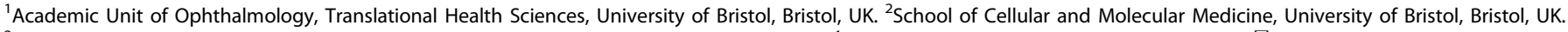
${ }^{3} \mathrm{NIHR}$ Biomedical Research Centre of Ophthalmology, Moorfields Eye Hospital, London, UK. ${ }^{4}$ UCL Institute of Ophthalmology, London, UK. ${ }^{\circledR e m a i l: ~ a . d i c k @ b r i s t o l . a c . u k ~}$ 
deposits between the Bruch's membrane and photoreceptors, the RPE is central in the maintenance of the visual cycle, and photoreceptor health [17, 19]. As such, RPE is metabolically demanding and mitochondria rich [20]. Through high-turnover phagocytosis of the outer segment and photo-stress, RPE mitochondrial respiration serves a major source of endogenous reactive oxygen species (ROS), which in turn and with age may lead to damage of mitochondria and the cellular integrity $[17,20]$. Indeed, metabolomics analyses of RPE from human AMD donors demonstrate significantly aberrant mitochondrial metabolites [21]. Whilst healthy RPE rely on antioxidative defences, including autophagy/mitophagy [20], iPS-RPE-derived cells from AMD patients, or with known risk mutations have reduced antioxidative stress response and produce higher levels of ROS [22, 23]. Defective RPE metabolism that associates with a low-grade unresolved inflammation, is considered as a major trigger event for AMD progression.

Metabolic dysfunction and oxidative stress are highly integrated with immune function within the tissue microenvironment (metabolic ecosystem) and in individual cells [5, 19, 20, 24, 25]. Excessive inflammatory mediators, such as cytokines, ROS, and nitric oxide, undermine mitochondrial function by inducing mitochondria DNA (mtDNA) mutations and inhibiting the mitochondrial respiratory chain for energy production [6, 17]. Concurrently, lipids, proteins, and DNA damaged by oxidation found in drusen and RPE are proinflammatory $[20,26]$. These tissue stressors accumulate with age and induce a parainflammatory response [27], which plays a protective role for the retina, for example driving phagocytosis of the drusen [28]. However, in AMD, a notion is that homeostatic para-inflammation itself becomes dysregulated and drives detrimental inflammation possibly due to excessive damage (e.g. from age or environmental factors) or genetic perturbation affecting the normal immune response (e.g. complement pathway risk alleles) [27, 29]. In this instance, a host of proinflammatory cytokines secreted from RPE, microglia, and macrophages, along with degranulation of choroidal mast cells [30-32] promote a proatrophic and proangiogenic environment for AMD progression [5, 33-35]. Overcoming such immune dysregulation, is a key therapeutic target. For example, eliciting conditions that promote a regulatory proresolution inflammatory phenotype has been shown to be antiangiogenic in experimental autoimmune uveitis and laser-induced CNV, respectively [5, 36, 37], and could have anti-inflammatory potential in AMD. Moreover, whilst diverse immune regulators, such as IL-10 and IL-4 are central for the immune homeostasis; there is increasing evidence that manifests their indispensable roles in also regulating cellular metabolism and maintaining cell health $[38,39]$. Indeed, metabolic programming of cells is distinct depending on the immune stimulator with IL-4 inducing an M2 skew in macrophages and commitment to oxidative phosphorylation (OXPHOS), whilst lipopolysaccharide (LPS) promotes a switch to aerobic glycolysis [40]. Moreover, metabolism and metabolites can directly influence the function and differentiation of immune cells (reviewed in $[40,41])$. In this review, we will cover the immunomodulatory potential of homeostatic cytokine interleukin-33 (IL-33), and the growing evidence supporting its therapeutic potential to target aspects of early metabolic and immune dysregulation in AMD.

\section{INTERLEUKIN-33 MODULATION OF DISEASE}

$\mathrm{IL}-33$ is a nuclear cytokine of the IL-1 family, which is constitutively and strongly expressed in epithelial, endothelial, and fibroblasts of barrier tissues [42-44]. IL-33 is considered an alarm signal ("alarmin"), a first-line defence responding to both endogenous and exogenous danger signals, such as tissue damage or allergen exposure [44, 45]. However, constitutive IL33 expression in cell types, such as adipocytes, glial cells, and neurons [44, 46-48], suggest a broader role for this cytokine beyond alarmin inflammatory function, highlighting potential roles in cellular and tissue homeostasis. Unlike the other IL-1 cytokine family members, IL-33 lacks a classical signal peptide for secretion from cells and is bioactive at full length. Thus, the effect of a "surge" IL-33 release from cells is best understood in conditions of allergy, necrosis, or mechanical injury [42]. Although, IL-33 is also secreted without cell death, in response to cell stressors, such as oxidative stress or increase of extracellular ATP [49-51], the mechanism of release remains elusive. Once secreted, IL-33 activity can be enhanced by proteases, in the context of acute or subacute inflammatory responses, from immune cells or allergens [42] or suppressed by soluble ST2 (decoy receptor) $[52,53]$ and caspase-dependent proteolysis [54]. Signalling via the ST2 receptor (IL-1RL1R) [42], IL33 has a wide range of cellular targets, including innate lymphoid cells 2 (ILC2s) [42, 55], mast cells [56], T-helper (Th)2 cells [42], macrophages [57], eosinophils as well all Th1, and natural killer cells $[42,58]$. As such, IL-33 plays a critical immune-modulatory role in allergic and chronic inflammatory and degenerative diseases, from asthma and cardiovascular disease to Alzheimer's disease [44, 59-61].

As an "alarmin" and strong inducer of Th2 immune responses, IL-33 is implicated in Th2-mediated allergic inflammatory disorders, such as asthma [44]. In lung biopsies of asthmatic patients, IL-33 expression is increased [62] and IL-33 polymorphisms can increase asthma susceptibility $[63,64]$. This is supported by observations in mice, where overexpression or exogenous administration of IL-33 promotes airway inflammation, whilst blocking the IL-33-ST2 axis reduces inflammation $[44,65]$. In this context, IL-33-ST2 signalling mediates both innate and adaptive Th2 responses in asthma, including eosinophils, ICL2s, Th2 cells, and mast cells [44], inducing secretion of proinflammatory cytokines and cellular degranulation. However, IL-33-stimulated mast cells are also important for balancing immune homeostasis in models of asthma, promoting the expansion of Treg cells [66], and protecting against the development of airway hyperresponsiveness [67]. Given these opposing regulatory roles, it may be the aberrant expression and activity of IL-33, exacerbated by allergens [42], that promotes dysregulation and subsequent pathological chronic inflammation. Certainly, under acute phototoxic stress in the retina, Müller glia cells secreted supraphysiological amounts of IL-33 into the vitreous leading to cell death and retina thinning [46]. Conversely, in the absence of provoking an acute release with or without aggravating exogenous environmental cues, IL-33 can adopt an important protective role, modulating the immune response and resolving inflammation in metabolic and autoimmune disorders, as well as mechanical injury $[36,57,68-70]$.

Patients with chronic heart failure have a reduced IL-33/sST2 ratio, suggesting an importance for IL-33 bioactivity, which is hindered by inhibitory SST2 [71]. Indeed, in mice, IL-33-mediated expansion of IL-5 and -13 expressing Th2 cells protects against atherosclerosis by stimulating the production of ox-LDL antibodies [59]. Similarly, in diet-induced obese mice, IL-33 expansion of ST2+ Treg cells reduces inflammation in visceral adipose tissue ameliorating insulin resistance [70], and in the eye retinal inflammation in the experimental autoimmune uveitis (EAU) model is attenuated by IL-33 expansion of Th2 cells and reduction of pathogenic Th17 inflammatory cells [36]. Interestingly, in the experimental autoimmune encephalomyelitis (EAE) model IL-33 can attenuate expansion of Th17 cells through mast cell-mediated alternative activation of macrophages (M2 skew), suggesting mast cells could play an important intermediary role in mediating protective effects of IL-33 [72]. In the eye, we have demonstrated that infiltration of tryptase + mast cells at lesion sites in the laserinduced choroidal neovascularisation (L-CNV) model, similar with both vehicle and IL-33 treatment [73]. Furthermore, co-culture 

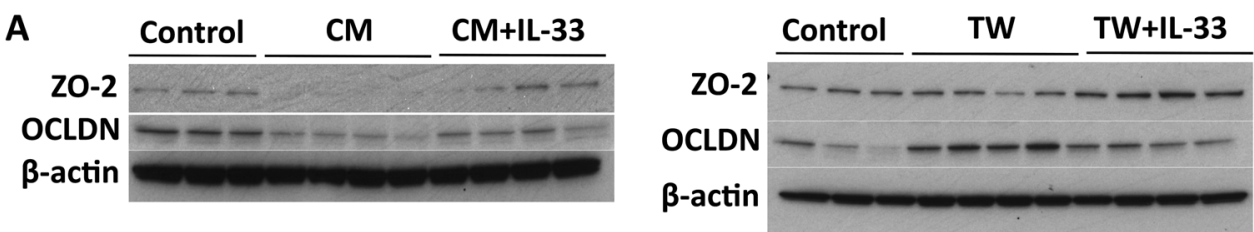

B
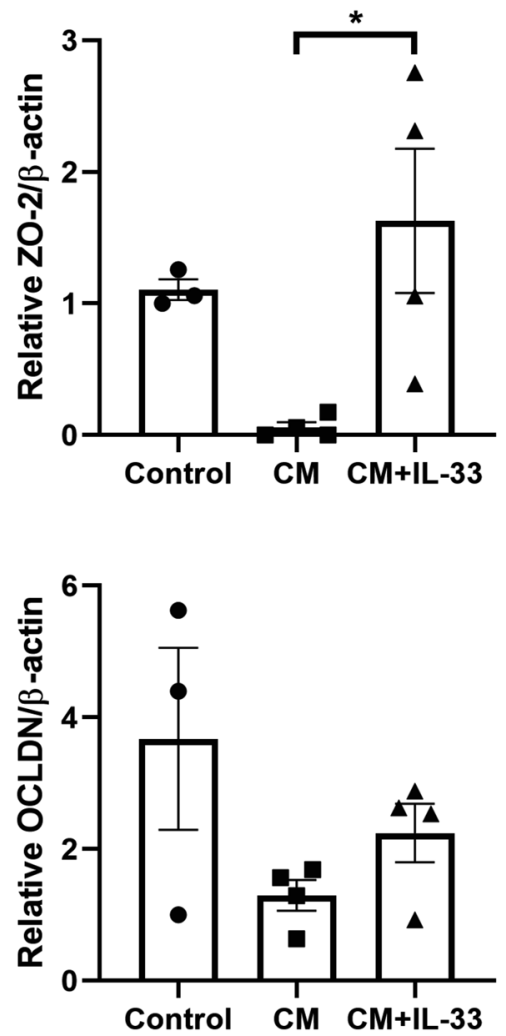
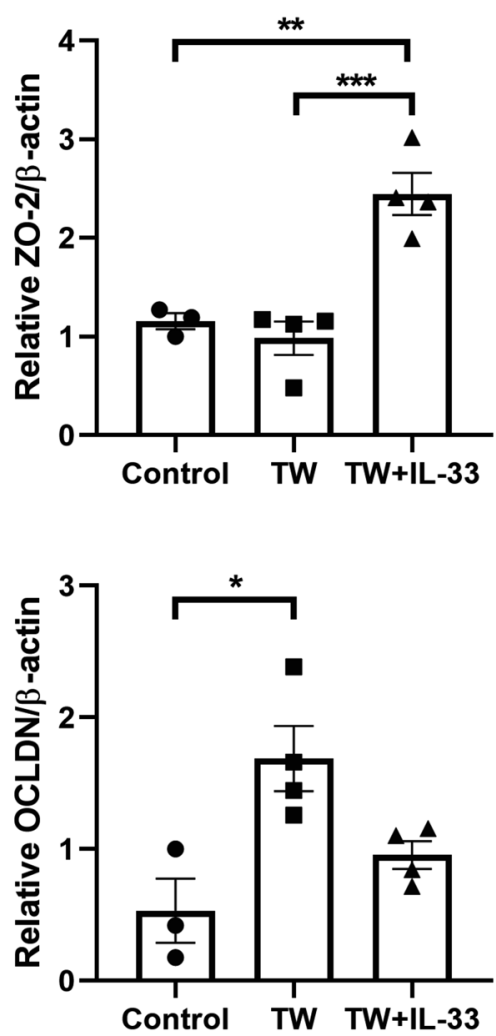

Fig. 1 IL-33 stimulated mast cells regulate tight-junction protein expression in RPE. A Western blot shows ZO-2, Occludin and beta-actin expression in B6RPE-07 lysates (mouse RPE cell line, courtesy of $\mathrm{H}$ Xu, Belfast) either cultured in standard media (control), in conditioned media (CM) from bone-marrow-derived mast cells with or without IL-33 stimulation (12 h), or co-cultured by transwell (TW) with treated or untreated mast cells. B Graphs show relative expression by densitometry analysis ${ }^{*} p<0.05,{ }^{* *} p<0.01$; one-way ANOVA with Tukey's multiple comparisons test).

with bone-marrow-derived mast cells or the conditioned media from the cells had a significant effect on the RPE cell expression of tight-junction proteins, zona occludins 2 (ZO-2), and occludin (OCLDN), when primed with IL-33 compared to unstimulated cells (Fig. 1). Changes to tight-junction proteins, including ZO-2 and OCLDN, are important in wound-healing responses and RPE barrier integrity and function [74], and therefore the IL-33mediated response of mast cell function could influence the outcome in this context. Furthermore, the importance of IL-33 in resolving inflammation is highlighted in a study by Augustine et al. that shows following retinal detachment, IL-33 deficient mice display chronic inflammatory responses and increased severity of retinal degeneration [68]. As in EAE, IL-33-mediated alternative activation of macrophages also plays an important role in promoting recovery after a spinal cord injury [57]. Whilst in the brain, IL-33-stimulated macrophage M2 phenotype of microglia is both key to maintaining synaptic plasticity, protecting against cognitive decline [47], as well as stimulating clearance of plaques in Alzheimer's models [60, 75]. The importance of IL-33 in modulating immune responses across diseases settings, highlights the homeostatic potential for this cytokine. However, the broad constitutive expression of IL-33 across cell types, including neurons, also suggests a wider role outside of immune regulation.
In brain tissue from Alzheimer's patients, IL-33 expression is decreased, and overexpression of nuclear IL-33 in vitro reduces the secretion of Amyloid-beta (AB) [40] peptides, independent of ST2 signalling [76]. Whilst it is recognised that IL-33 stimulation of macrophages is important for clearing $A \beta$ plaques $[60,75]$, other reports demonstrate that IL-33 loss impairs the autophagic clearance of cell waste in neurons and leads to furthers neuronal loss [48]. In ovarian tissue, loss of IL-33 resulted in the loss of autophagic function, subsequent waste accumulation and tissue degeneration [77]. Together, these data strongly infer a critical role for IL-33 in maintaining autophagy and thus cell heath and tissue function across a variety of tissues.

IL-33 stimulation also has a direct effect on cell metabolism, increasing glycolysis and OXPHOS in mast cells [78] and reshaping metabolism in macrophages [79]. Our recent work further uncovered IL-33/ST2-mediated changes to metabolism in RPE cells but importantly revealed a novel role for nuclear IL-33 as a critical metabolic regulator (discussed in the next section) [80]. Thus, these findings and emerging evidence supports a broader role for IL-33, through both the IL-33/ST2 signalling axis and within the nucleus. Moreover, with a homeostatic role in immune modulation, metabolism and autophagy, the therapeutic potential for IL-33 is extensive and far reaching, in 
particular, for tissues with a high metabolic load, such as the CNS and retina.

\section{THERAPEUTIC DEVELOPMENT AND THE POTENTIAL OF IL-33 FOR TREATING AAMD}

The complexity of AMD pathogenesis has led to several approaches for therapeutic development, targeting the different pathways that contribute to disease progression. The discovery of complement associated risk variants combined with the identification of complement components in drusen [81], has driven a multitude of the investigation of complement pathway inhibitors as potential AMD therapeutics, with mixed results [82]. Many approaches investigated follow a paradigm of intravitreal injections, which still impacts patient compliance and ability to deliver at scale in healthcare settings. As such, complement inhibition and anti-inflammatory therapies are expanding into gene therapy approaches. The pipeline of gene therapy for ophthalmic eye disorders is expanding, with successes in inherited retinal degenerative disorders [83] and the potential for a "one-hit" treatment approach for AMD may provide great appeal.

Due to the importance of RPE in maintaining the retina and photoreceptor health and the contribution of RPE oxidative stress to pathological progression of AMD, another therapeutic strategy is antioxidant supplement treatment and mitochondrial enhancer, Elamipretide, with some success in early trials [82]. Certainly, the potential for gene augmentation of oxidative stress modulators is being investigated further and bring an opportunity to drive additional candidate targets for gene therapy in AMD. The push towards identifying gene therapy targets for AMD is a promising avenue for treatment, however; due to AMD complexity identifying the right candidate is essential for reaching the wider aAMD patient population. Considering the convergence of affected pathways, autophagy/mitophagy, oxidative stress/metabolism and inflammation, the efficacy of gene therapy in AMD will be maximised by the identification of a cross-regulatory target.

In our recent work, we illuminated a novel role for nuclear expressed IL-33 as a key regulator of metabolism in RPE cells. The nuclear expression of IL-33 is essential for utilisation of pyruvate in the TCA cycle and mitochondrial metabolism, through inducing the abundance of mitochondrial pyruvate carrier 1 (MPC1). Primary RPE cells from IL-33-/- mice have significant changes to mitochondria structure compared to wild-type cells, displaying irregularity in size and increased short tubular phenotypes [80]. Similar mitochondrial changes were observed in human RPE cells from donors $>60$ years in age compared to young donors and correlated with a reduction in antioxidant defences [84] and IL-33 expression (see below), consolidating the importance of IL-33 expression for mitochondrial structure and metabolism. Conversely, IL-33 antagonises aerobic glycolysis, so that with loss of IL-33 expression cells exhibit increased extracellular acidification rate (ECAR) and extracellular lactate [80]. Reduction or loss of IL-33 in RPE could lead to devastating changes to the outer retinal homeostasis, particularly if RPE adopt aerobic glycolysis over mitochondria respiration $[85,86]$ and yield RPE vulnerable to oxidative stress, conditions permissive for AMD pathogenesis. Indeed, the data-mining approach of published transcriptomic datasets reveals that the RPE/choroid/sclera tissues of AMD patients have decreased IL-33 mRNA expression compared to normal age-matched donors, whilst expression is unchanged in the retina (Fig. 2A, B (ref [87])). In support, and specific to the RPE, we see a reduction in IL-33 protein expression and concurrent RPE damage in a mouse model of light-induced retinal damage, emulating oxidative stress (unpublished, Fig. 2C-E). The nuclear importance of IL-33 to the RPE metabolic phenotype is distinct from the IL-33/ST2 signalling, as evidenced by the lack of metabolic phenotype in IL1rl1-/- mice [80]. However, in parallel we find IL-33 signalling via ST2 also contributes to promoting oxidative phosphorylation and mitochondrial respiration in RPE cells, through activation of AMP-activated protein kinase (AMPK). Importantly, treatment of RPE cells with recombinant IL-33 protects against $\mathrm{H}_{2} \mathrm{O}_{2}$ induced oxidative stress [80], suggesting IL-33/ST2 signalling could have therapeutic potential for RPE in AMD. Accordingly, we have shown IL-33 delivery in the aged $\mathrm{Cf}+\mathrm{I}-$ high-fat diet-fed model of outer retinal degeneration can protect against RPE loss and maintain expression of key metabolic proteins in the retina [88]. Additional evidence shows that stimulation of AMPK by metformin protects against photoreceptor
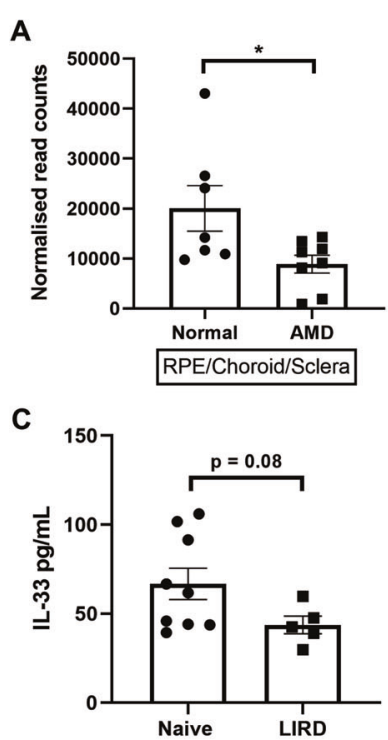

B

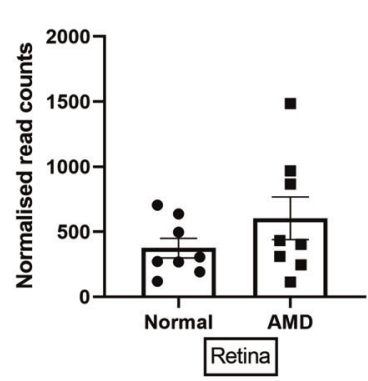

D

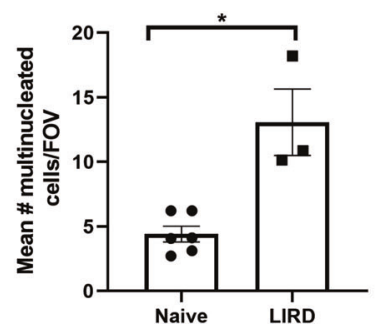

E

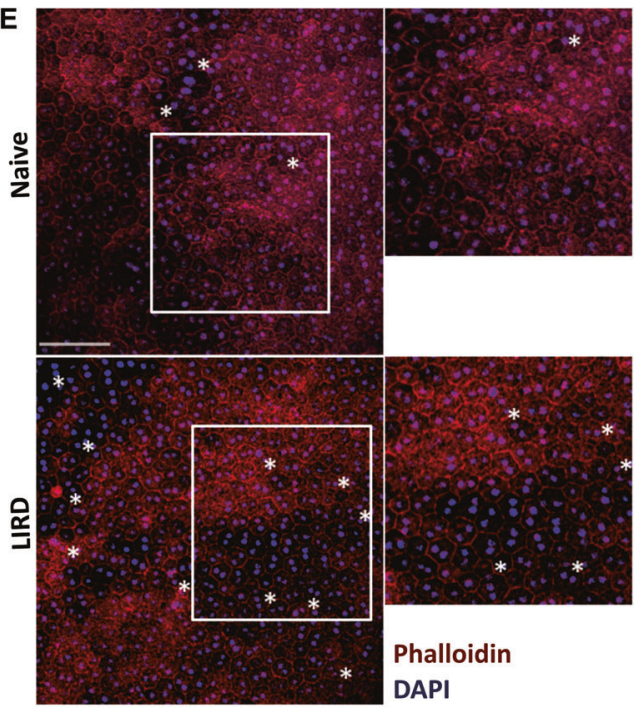

Fig. 2 Association of altered IL-33 expression with AMD and RPE damage. IL-33 gene expression is decreased in RPE/choroid/sclera of AMD donors by more than $50 \%$ compared to normal age-matched donors ( $p=0.03$, two-tailed t-test) $\mathbf{A}$ but expression is unchanged in the retina $\mathbf{B}$. RNAsequencing data from Kim et al. (GSE99248). C ELISA analysis shows a reduction of IL-33 protein in RPE lysates from a mouse model of light-induced retinal damage (100k LUK/20 min), compared to naïve eyes $(p=0.08$, Mann-Whitney $U$ test). D Concurrently, RPE damage is apparent, indicated by an increase in the number of multinucleated cells (RPE cells containing $\geq 3$ nuclei/cell within the cell boundary; ${ }^{*} p=0.02$, Mann-Whitney $U$ test), counted using ImageJ. E Representative images of RPE/choroid flatmounts, stained with phalloidin-Alexa555 and DAPI. Multinucleated cells are indicated $(*)$. Scale bar is $80 \mu \mathrm{m}$. 


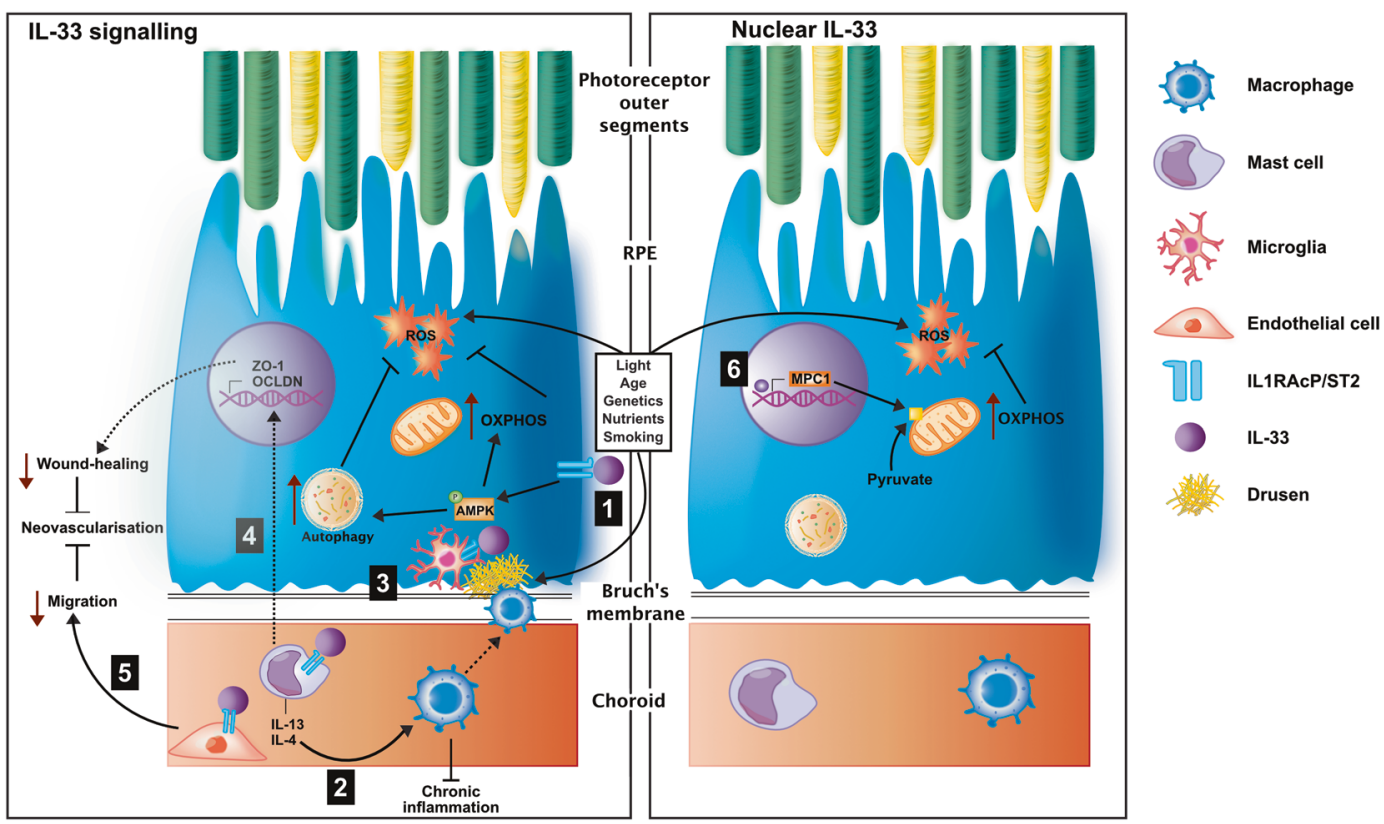

Fig. 3 IL-33 therapeutic potential in early AMD. The interleukin-33 biological function has The potential to protect against early pathogenic pathways in AMD via dual function, as a signalling molecule and nuclear factor. With diverse cellular targets, IL-33 can stimulate 1) retinal pigment epithelium (RPE) in the eye directly, increasing AMPK phosphorylation and subsequent mitochondrial metabolism ${ }^{78}$. AMPK phosphorylation can also increase autophagy. Both will combat reactive oxygen species (ROS) production and oxidative stress. 2) IL-33 influences alternative activation of macrophages by stimulation of mast cells to release IL-13 and IL-4 ${ }^{68}$, which can promote inflammation resolution. 3) IL-33 stimulation of an alternative microglia phenotype promotes deposit clearance in Alzheimer's disease models ${ }^{59,73}$ and could prove beneficial against drusen. 4) IL-33 stimulated mast cells to induce changes to tight-junction proteins in RPE cells 5) and directly stimulate retinal endothelial cells reducing migration ${ }^{71}$, both important in mediating wound-healing responses. 6) Nuclear IL-33 is a key metabolic regulator in RPE cells, promoting increased mitochondrial metabolism (OXPHOS; oxidative phosphorylation) ${ }^{78}$. The dashed line indicates indirect evidence.

and RPE damage in retinal degeneration models, including lightinduced damage [89], and that activation of AMPK converges on pathways promoting autophagy [90]. IL-33 signalling could therefore also have beneficial effects for autophagy in RPE cells, another key target for combating pathogenic changes in AMD. Loss of IL-33 in the brain and ovarian follicle tissues leads to a reduction in a central autophagy protein, LC3II, supporting an important role for IL-33 in maintaining autophagy [48, 77]. Collectively, through distinct mechanisms, IL-33 has potential both as a nuclear effector and by canonical IL-33/ST2 signalling to maintain and promote metabolic homeostasis and autophagy in RPE. Therapeutically targeting converging metabolic and autophagic pathways brings an opportunity to ameliorate disease progression in the early stages of aAMD.

Further, in $10-15 \%$ of cases, AMD will progress to neovascularisation, driven by aberrant angiogenesis and fibrosis [91]. We have demonstrated that treatment of human choroidal fibroblasts with recombinant IL-33 reduces expression of MMPs (2/9), stalling cell migration, and the wound-healing responses behind fibrosis. Additionally, in a mouse model of choroidal neovascularisation, we found IL-33/ST2 signalling from recombinant IL-33 is antiangiogenic and reduces neovascular lesion volume [73]. Treating early in AMD with IL-33 could therefore not only be beneficial in targeting disease pathways of aAMD but also provide protection against advancement to neovascularisation. Together, evidence suggests IL-33 treatment early in AMD may protect RPE, promote metabolic and autophagic homeostasis, and modulate infiltrating cells, resolving pathogenic immune dysregulation and angiogenesis (Fig. 3).

\section{CONCLUSIONS}

The complex multifactorial nature of early and aAMD pathogenesis provides a huge challenge in developing effective therapeutics to benefit a wide population of patients. Advancements in understanding the disease pathways and risk alleles behind AMD has supported the development of several targeted therapeutic approaches. However, identifying a target that can tackle multiple pathways of aAMD pathogenesis remains critical. Growing evidence of the homeostatic nature for cytokine IL-33, mediating metabolism, autophagy, and modulating the immune response, provides the exciting potential to target the converging disease pathways of AMD. Future work in models emulating disease pathways of aAMD will be important to confirm beneficial effects for IL-33 in modulating immune dysregulation under disease conditions.

\section{REFERENCES}

1. Wong WL, Su X, Li X, Cheung CMG, Klein R, Cheng CY, et al. Global prevalence of age-related macular degeneration and disease burden projection for 2020 and 2040: a systematic review and meta-analysis. Lancet Glob Heal. 2014;2:e106-16.

2. Handa JT, Bowes Rickman C, Dick AD, Gorin MB, Miller JW, Toth CA, et al. A systems biology approach towards understanding and treating non-neovascular age-related macular degeneration. Nat Commun. 2019;10:1-11.

3. Chen $\mathrm{M}, \mathrm{Xu} \mathrm{H}$. Parainflammation, chronic inflammation, and age-related macular degeneration. J Leukoc Biol. 2015;98:713-25.

4. Pool FM, Kiel C, Serrano L, Luthert PJ. Repository of proposed pathways and protein-protein interaction networks in age-related macular degeneration. NPJ Aging Mech Dis 2020;6:2.

5. Copland DA, Theodoropoulou S, Liu J, Dick AD. A perspective of AMD through the eyes of immunology. Investig Ophthalmol Vis Sci. 2018;59:83-92.

6. Lee KS, Lin S, Copland DA, Dick AD, Liu J. Cellular senescence in the aging retina and developments of senotherapies for age-related macular degeneration. J Neuroinflammation. 2021;18:32.

7. Heesterbeek TJ, Lorés-Motta L, Hoyng CB, Lechanteur YTE, den Hollander AI. Risk factors for progression of age-related macular degeneration. Ophthalmic Physiol Opt. 2020;40:140-70.

8. Fritsche LG, Igl W, Bailey JNC, Grassmann F, Sengupta S, Bragg-Gresham JL, et al. A large genome-wide association study of age-related macular 
degeneration highlights contributions of rare and common variants. Nat Genet. 2016;48:134-43.

9. Fritsche LG, Chen W, Schu M, Yaspan BL, Yu Y, Thorleifsson G, et al. Seven new loci associated with age-related macular degeneration. Nat Genet. 2013;45:433-9.

10. Winkler TW, Grassmann F, Brandl C, Kiel C, Günther F, Strunz T, et al. Genomewide association meta-analysis for early age-related macular degeneration highlights novel loci and insights for advanced disease. BMC Med Genomics. 2020;13:1-18.

11. Yaspan BL, Williams DF, Holz FG, Regillo CD, Li Z, Dressen A, et al. Targeting factor $D$ of the alternative complement pathway reduces geographic atrophy progression secondary to age-related macular degeneration. Sci Transl Med. 2017;9: eaaf1443.

12. Holz FG, Sadda SR, Busbee B, Chew EY, Mitchell P, Tufail A, et al. Efficacy and safety of Lampalizumab for geographic atrophy due to age-related macular degeneration. JAMA Ophthalmol. 2018;136:666-77.

13. Dreismann AK, McClements ME, Barnard AR, Orhan E, Hughes JP, Lachmann PJ, et al. Functional expression of complement factor I following AAV-mediated gene delivery in the retina of mice and human cells. Gene Ther 2021;28:265-76.

14. Rohrer B, Coughlin B, Kunchithapautham K, Long Q, Tomlinson S, Takahashi K, et al. The alternative pathway is required, but not alone sufficient, for retinal pathology in mouse laser-induced choroidal neovascularization. Mol Immunol. 2011;48:e1-8.

15. Ding JD, Kelly U, Landowski M, Toomey CB, Groelle M, Miller C, et al. Expression of human complement factor $\mathrm{h}$ prevents age-related macular degeneration-like retina damage and kidney abnormalities in aged cfh knockout mice. Am J Pathol. 2015;185:29-42.

16. Toomey CB, Landowski M, Klingeborn M, Kelly U, Deans J, Dong $H$, et al. Effect of anti-C5a therapy in a murine model of early/ intermediate dry age-related macular degeneration. Investig Ophthalmol Vis Sci. 2018;59:662-73.

17. Kaarniranta K, Uusitalo $H$, Blasiak J, Felszeghy S, Kannan R, Kauppinen A, et al. Mechanisms of mitochondrial dysfunction and their impact on age-related macular degeneration. Prog Retin Eye Res. 2020;79:100858.

18. Lim LS, Mitchell P, Seddon JM, Holz FG, Wong TY. Age-related macular degeneration. Lancet. 2012;379:1728-38.

19. Rozing MP, Durhuus JA, Krogh Nielsen M, Subhi Y, Kirkwood TB, Westendorp RG, et al. Age-related macular degeneration: a two-level model hypothesis. Prog Retin Eye Res. 2020;76:100825.

20. Datta S, Cano M, Ebrahimi K, Wang L, Handa JT. The impact of oxidative stress and inflammation on RPE degeneration in non-neovascular AMD. Prog Retin Eye Res. 2017;60:201-18.

21. Brown CN, Green BD, Thompson RB, Den Hollander Al, Lengyel I. Metabolomics and age-related macular degeneration. Metabolites. 2019;9:4

22. Yang J, Li Y, Chan L, Tsai YT, Wu WH, Nguyen HV, et al. Validation of genome-wide association study (GWAS)-identified disease risk alleles with patient-specific stem cell lines. Hum Mol Genet. 2014;23:3445-55.

23. Golestaneh N, Chu Y, Cheng SK, Cao H, Poliakov E, Berinstein DM. Repressed sirt1/ pgc-1a pathway and mitochondrial disintegration in ipsc-derived rpe disease model of age-related macular degeneration. J Transl Med. 2016;14:1-17.

24. Liu J, Copland DA, Theodoropoulou S, Chiu HAA, Barba MD, Mak KW, et al. Impairing autophagy in retinal pigment epithelium leads to inflammasome activation and enhanced macrophage-mediated angiogenesis. Sci Rep. 2016;6:1-15.

25. Curcio $C A$, Johnson $M$, Rudolf $M$, Huang JD. The oil spill in ageing Bruch membrane. Br J Ophthalmol. 2011;95:1638-45.

26. Chou MY, Hartvigsen K, Hansen LF, Fogelstrand L, Shaw PX, Boullier A, et al Oxidation-specific epitopes are important targets of innate immunity. J Intern Med. 2008;263:479-88.

27. Xu H, Chen M, Forrester JV. Para-inflammation in the aging retina. Prog Retin Eye Res 2009;28:348-68.

28. Guillonneau X, Eandi CM, Paques M, Sahel JA, Sapieha P, Sennlaub F. On phagocytes and macular degeneration. Prog Retin Eye Res. 2017;61:98-128.

29. Weismann D, Hartvigsen K, Lauer N, Bennett KL, Scholl HPN, Issa PC, et al. Complement factor $\mathrm{H}$ binds malondialdehyde epitopes and protects from oxidative stress. Nature. 2011;478:76-81.

30. Ogura S, Baldeosingh R, Bhutto IA, Kambhampati SP, Scott McLeod D, Edwards MM, et al. A role for mast cells in geographic atrophy. FASEB J 2020;34:10117-31.

31. McLeod DS, Bhutto I, Edwards MM, Gedam M, Baldeosingh R, Lutty GA. Mast cellderived tryptase in geographic atrophy. Investig Ophthalmol Vis Sci. 2017;58:5887-96.

32. Bhutto IA, McLeod DS, Jing T, Sunness JS, Seddon JM, Lutty GA. Increased choroidal mast cells and their degranulation in age-related macular degeneration. Br J Ophthalmol. 2016;100:720-6.

33. Shaw PX, Zhang L, Zhang M, Du H, Zhao L, Lee C, et al. Complement factor $H$ genotypes impact risk of age-related macular degeneration by interaction with oxidized phospholipids. Proc Natl Acad Sci USA. 2012;109:13757-62.

34. Marazita MC, Dugour A, Marquioni-Ramella MD, Figueroa JM, Suburo AM. Oxidative stress-induced premature senescence dysregulates VEGF and CFH expression in retinal pigment epithelial cells: implications for age-related macular degeneration Redox Biol. 2016;7:78-87.

35. Rashid K, Akhtar-Schaefer I, Langmann T. Microglia in retinal degeneration. Front Immunol 2019;10:1975

36. Barbour M, Allan D, Xu H, Pei C, Chen M, Niedbala W, et al. IL-33 attenuates the development of experimental autoimmune uveitis. Eur J Immunol 2014;44:3320-9.

37. Wu WK, Georgiadis A, Copland DA, Liyanage S, Luhmann UFO, Robbie SJ, et al. IL-4 regulates specific Arg-1+ macrophage sFlt-1-mediated inhibition of angiogenesis. Am J Pathol. 2015;185:2324-35.

38. Ip WKE, Hoshi N, Shouval DS, Snapper S, Medzhitov R. Anti-inflammatory effect of IL-10 mediated by metabolic reprogramming of macrophages. Science. 2017;356:513-9.

39. Brestoff JR, Artis D. Immune regulation of metabolic homeostasis in health and disease. Cell. 2015;161:146-60.

40. O'Neill LAJ, Pearce EJ. Immunometabolism governs dendritic cell and macrophage function. J Exp Med. 2016;213:15-23.

41. Patel $\mathrm{CH}$, Leone RD, Horton MR, Powell JD. Targeting metabolism to regulate immune responses in autoimmunity and cancer. Nat Rev Drug Disco. 2019;18 669-88.

42. Cayrol C, Girard JP. Interleukin-33 (IL-33): a nuclear cytokine from the IL-1 family. Immunol Rev. 2018;281:154-68.

43. Chan BCL, Lam CWK, Tam LS, Wong CK. IL33: roles in allergic inflammation and therapeutic perspectives. Front Immunol. 2019;10:364.

44. Ding W, Zou GL, Zhang W, Lai XN, Chen HW, Xiong LX. Interleukin-33: its emerging role in allergic diseases. Molecules. 2018;23:1665.

45. Martin NT, Martin MU. Interleukin 33 is a guardian of barriers and a local alarmin. Nat Immunol. 2016;17:122-31.

46. Xi H, Katschke KJ, Li Y, Truong T, Lee WP, Diehl L, et al. IL-33 amplifies an innate immune response in the degenerating retina. J Exp Med. 2016;213:189-207.

47. Nguyen PT, Dorman LC, Pan S, Vainchtein ID, Han RT, Nakao-Inoue H, et al. Microglial remodeling of the extracellular matrix promotes synapse plasticity. Cell. 2020;182:388-403.e15.

48. Carlock C, Wu J, Shim J, Moreno-Gonzalez I, Pitcher MR, Hicks J, et al. Interleukin33 deficiency causes tau abnormality and neurodegeneration with Alzheimer-like symptoms in aged mice. Transl Psychiatry. 2017;7:e1164.

49. Uchida M, Anderson EL, Squillace DL, Patil N, Maniak PJ, lijima K, et al. Oxidative stress serves as a key checkpoint for IL-33 release by airway epithelium. Allergy Eur. J Allergy Clin Immunol. 2017;72:1521-31.

50. Kouzaki H, lijima K, Kobayashi T, O'Grady SM, Kita H. The danger signal, extracellular ATP, is a sensor for an airborne allergen and triggers IL-33 release and innate Th2-type responses. J Immunol. 2011;186:4375-87.

51. Hristova $M$, Habibovic A, Veith $C$, Janssen-Heininger YMW, Dixon AE, Geiszt $M$ et al. Airway epithelial dual oxidase 1 mediates allergen-induced IL-33 secretion and activation of type 2 immune responses. J Allergy Clin Immunol. 2016;137: 1545-1556.e11.

52. Hayakawa H, Hayakawa M, Kume A, Tominaga SI. Soluble ST2 blocks interleukin-33 signaling in allergic airway inflammation. J Biol Chem. 2007;282:26369-80.

53. Bandara G, Beaven MA, Olivera A, Gilfillan AM, Metcalfe DD. Activated mast cells synthesize and release soluble ST2-a decoy receptor for IL-33. Eur J Immunol. 2015;45:3034-44.

54. Lüthi AU, Cullen SP, McNeela EA, Duriez PJ, Afonina IS, Sheridan C, et al. Suppression of interleukin-33 bioactivity through proteolysis by apoptotic caspases. Immunity. 2009;31:84-98.

55. Mjösberg JM, Trifari S, Crellin NK, Peters CP, Van Drunen CM, Piet B, et al. Human IL-25-and IL-33-responsive type 2 innate lymphoid cells are defined by expression of CRTH2 and CD161. Nat Immunol. 2011;12:1055-62.

56. Enoksson M, Lyberg K, Möller-Westerberg C, Fallon PG, Nilsson G, LunderiusAndersson C. Mast cells as sensors of cell injury through IL-33 recognition. J Immunol. 2011;186:2523-8.

57. Gadani SP, Walsh JT, Smirnov I, Zheng J, Kipnis J. The Glia-derived alarmin IL-33 orchestrates the immune response and promotes recovery following CNS injury. Neuron. 2015;85:703-9.

58. Molofsky AB, Savage AK, Locksley RM. Interleukin-33 in tissue homeostasis, injury, and inflammation. Immunity. 2015;42:1005-19.

59. Miller AM, Xu D, Asquith DL, Denby L, Li Y, Sattar N, et al. IL-33 reduces the development of atherosclerosis. J Exp Med. 2008;205:339-46.

60. Fu AKY, Hung KW, Yuen MYF, Zhou X, Mak DSY, Chan ICW, et al. IL-33 ameliorates Alzheimer's disease-like pathology and cognitive decline. Proc Natl Acad Sci USA. 2016;113:E2705-13.

61. Kurowska-Stolarska M, Kewin P, Murphy G, Russo RC, Stolarski B, Garcia CC, et al. IL-33 induces antigen-specific IL-5 + T cells and promotes allergic-induced airway inflammation independent of IL-4. J Immunol. 2008;181:4780-90.

62. Préfontaine $D$, Lajoie-Kadoch $S$, Foley $S$, Audusseau $S$, Olivenstein $R$, Halayko AJ, et al. Increased expression of IL-33 in severe asthma: evidence of expression by airway smooth muscle cells. J Immunol. 2009;183:5094-103. 
63. Savenije OE, Mahachie John JM, Granell R, Kerkhof M, Dijk FN, De Jongste JC, et al. Association of IL33-IL-1 receptor-like 1 (IL1RL1) pathway polymorphisms with wheezing phenotypes and asthma in childhood. J Allergy Clin Immunol. 2014;134:170-7.

64. Schröder PC, Casaca VI, Illi S, Schieck M, Michel S, Böck A, et al. IL-33 polymorphisms are associated with increased risk of hay fever and reduced regulatory T cells in a birth cohort. Pediatr Allergy Immunol. 2016;27: 687-95.

65. Miller AM. Role of IL-33 in inflammation and disease. J Inflamm. 2011;8:22.

66. Morita H, Arae K, Unno H, Miyauchi K, Toyama S, Nambu A, et al. An interleukin33-mast cell-interleukin-2 axis suppresses papain-induced allergic inflammation by promoting regulatory $T$ cell numbers. Immunity. 2015;43:175-86.

67. Zoltowska Nilsson AM, Lei Y, Adner M, Nilsson GP. Mast cell-dependent IL-33/ ST2 signaling is protective against the development of airway hyperresponsiveness in a house dust mite mouse model of asthma. Am J Physiol - Lung Cell Mol Physiol. 2018;314:L484-L492.

68. Augustine J, Pavlou S, Ali I, Harkin K, Ozaki E, Campbell M, et al. IL-33 deficiency causes persistent inflammation and severe neurodegeneration in retinal detachment. J Neuroinflammation. 2019;16:1-15.

69. Finlay CM, Cunningham KT, Doyle B, Mills KHG. IL-33-stimulated murine mast cells polarize alternatively activated macrophages, which suppress $T$ cells that mediate experimental autoimmune encephalomyelitis. J Immunol. 2020;205:1909-19.

70. Han JM, Wu D, Denroche HC, Yao Y, Verchere CB, Levings MK. IL-33 reverses an obesity-induced deficit in visceral adipose tissue ST2 $+\mathrm{T}$ regulatory cells and ameliorates adipose tissue inflammation and insulin resistance. J Immunol. 2015;194:4777-83.

71. Zhang HF, Xie SL, Chen YX, Mai JT, Wang JF, Zhu WL, et al. Altered serum levels of IL-33 in patients with advanced systolic chronic heart failure: correlation with oxidative stress. J Transl Med. 2012;10:120.

72. Jiang HR, Milovanović M, Allan D, Niedbala W, Besnard AG, Fukada SY, et al. IL-33 attenuates EAE by suppressing IL-17 and IFN- $\gamma$ production and inducing alternatively activated macrophages. Eur J Immunol. 2012;42:1804-14.

73. Theodoropoulou S, Copland DA, Liu J, Wu J, Gardner PJ, Ozaki E, et al. Interleukin33 regulates tissue remodelling and inhibits angiogenesis in the eye. J Pathol. 2017;241:45-56.

74. Leonardo TR, Shi J, Chen D, Trivedi HM, Chen L. Differential expression and function of bicellular tight junctions in skin and oral wound healing. Int J Mol Sci. 2020;21:1-17.

75. Lau SF, Chen C, Fu WY, Qu JY, Cheung TH, Fu AKY, et al. IL-33-PU.1 transcriptome reprogramming drives functional state transition and clearance activity of microglia in Alzheimer's disease. Cell Rep. 2020;31:107530.

76. Chapuis J, Hot D, Hansmannel F, Kerdraon O, Ferreira S, Hubans C, et al. Transcriptomic and genetic studies identify IL-33 as a candidate gene for Alzheimer's disease. Mol Psychiatry. 2009;14:1004-16.

77. Wu J, Carlock C, Zhou C, Nakae S, Hicks J, Adams HP, et al. IL-33 Is Required for disposal of unnecessary cells during ovarian atresia through regulation of autophagy and macrophage migration. J Immunol. 2015;194:2140-7.

78. Caslin $\mathrm{HL}$, Taruselli MT, Haque T, Pondicherry N, Baldwin EA, Barnstein BO, et al. Inhibiting glycolysis and ATP production attenuates IL-33-mediated mast cell function and peritonitis. Front Immunol. 2018;9:3026.

79. Xu H, Sun L, He Y, Yuan X, Niu J, Su J, et al. Deficiency in IL-33/ST2 axis reshapes mitochondrial metabolism in lipopolysaccharide-stimulated macrophages. Front Immunol. 2019;10:127.

80. Scott LM, Dick AD, Theodoropoulou S, Scott LM, Vincent EE, Hudson N, et al. Interleukin-33 regulates metabolic reprogramming of the retinal pigment epithelium in response to immune stressors Interleukin-33 regulates metabolic reprogramming of the retinal pigment epithelium in response to immune stressors. JCI Insight. 2021;6:e129429.

81. Kijlstra A, Berendschot TTJM. Age-related macular degeneration: a complementopathy? Ophthalmic Res. 2015;54:64-73.

82. Cabral De Guimaraes TA, Daich Varela M, Georgiou M, Michaelides M. Treatments for dry age-related macular degeneration: therapeutic avenues, clinical trials and future directions. Br J Ophthalmol. 2021. https://doi.org/10.1136/bjophthalmol2020-318452.

83. Wang X, Yu C, Tzekov RT, Zhu Y, Li W. The effect of human gene therapy for RPE65-associated Leber's congenital amaurosis on visual function: a systematic review and meta-analysis. Orphanet J Rare Dis. 2020;15:1-9.

84. He Y, Ge J, Burke JM, Myers RL, Dong ZZ, Tombran-Tink J. Mitochondria impairment correlates with increased sensitivity of aging RPE cells to oxidative stress. J Ocul Biol Dis Info. 2010;3:92-108.
85. Cheng SY, Cipi J, Ma S, Hafler BP, Kanadia RN, Brush RS, et al. Altered photoreceptor metabolism in mouse causes late stage age-related macular degeneration-like pathologies. Proc Natl Acad Sci USA. 2020;117:13094-104.

86. Petit L, Ma S, Cipi J, Cheng SY, Zieger M, Hay N, et al. Aerobic glycolysis is essential for normal rod function and controls secondary cone death in retinitis pigmentosa. Cell Rep. 2018;23:2629-42.

87. Kim EJ, Grant GR, Bowman AS, Haider N, Gudiseva HV, Chavali VRM. Complete transcriptome profiling of normal and age-related macular degeneration eye tissues reveals dysregulation of anti-sense transcription. Sci Rep.2018;8:1-13.

88. Clare AJ, Copland DA, Nicholson LB, Liu J, Neal CR, Moss S, et al. Treatment with interleukin-33 is non-toxic and protects retinal pigment epithelium in an ageing model of outer retinal degeneration. J Cell Mol Med. 2020;24:13546-50.

89. Xu L, Kong L, Wang J, Ash JD. Stimulation of AMPK prevents degeneration of photoreceptors and the retinal pigment epithelium. Proc Natl Acad Sci. 2018; 115:10475-80.

90. Jia J, Bissa B, Brecht L, Allers L, Choi SW, Gu Y, et al. AMPK, a regulator of metabolism and autophagy, Is activated by lysosomal damage via a novel galectin-directed ubiquitin signal transduction system. Mol Cell. 2020;77:951-969.e9.

91. Friedlander M. Fibrosis and diseases of the eye. J Clin Invest. 2007;117:576-86.

\section{ACKNOWLEDGEMENTS}

The authors would like to thank Dr Ying Kai Chan for their thoughts and comments on the review conception. Professional support for figure graphics was provided by Bio Sci Design.

\section{AUTHOR CONTRIBUTIONS}

A.C. wrote the manuscript and generated the figures. J.L., D.A.C., S.T., and A.D.D. wrote, reviewed, and edited the manuscript. All authors contributed to conceptualisation and approved the final version of the manuscript.

\section{FUNDING}

Data included in this review paper were supported by grants from the Academy of Medical Sciences (ST and ADD), GOAP Bayer award (ST) and Elizabeth Blackwell Institute Wellcome Trust (ADD).

\section{COMPETING INTERESTS}

ADD and ST are named inventors on UK patent applications 2015780.6 and 2015778.0.

\section{ADDITIONAL INFORMATION}

Correspondence and requests for materials should be addressed to A.D.D.

Reprints and permission information is available at http://www.nature.com/ reprints

Publisher's note Springer Nature remains neutral with regard to jurisdictional claims in published maps and institutional affiliations.

(i) Open Access This article is licensed under a Creative Commons Attribution 4.0 International License, which permits use, sharing, adaptation, distribution and reproduction in any medium or format, as long as you give appropriate credit to the original author(s) and the source, provide a link to the Creative Commons license, and indicate if changes were made. The images or other third party material in this article are included in the article's Creative Commons license, unless indicated otherwise in a credit line to the material. If material is not included in the article's Creative Commons license and your intended use is not permitted by statutory regulation or exceeds the permitted use, you will need to obtain permission directly from the copyright holder. To view a copy of this license, visit http://creativecommons. org/licenses/by/4.0/.

(c) The Author(s) 2021 